\title{
Light as a Building Matter of the Universe
}

\author{
Wim Vegt* \\ *Technical University, Eindhoven, The Netherlands \\ Correspondence: j.w.vegt@topacademy.center
}

The famous 1927 Solvay Conference was a turning point in Physics. Scientific realists, like Albert Einstein, had lost a fundamental conflict and instrumentalists, like Niels Bohr, had won. Since then, Physics has followed the path of instrumentalists in which the quantum physics has been determined by the concept of elementary particles and probability waves. Until May 2013, when for the first time a photo had been made of the probability waves inside the hydrogen atom, it was impossible to make a photo of a non-existing complex probability wave with mass zero, but the fact could not be denied anymore and scientists had succeeded to make the "complex" and "non-existing" quantum mechanical "probability wave" visible. New discoveries, like "photonic molecules" and "photon-photon interaction", require a new interpretation of the Copenhagen Interpretation. Since the Copenhagen Interpretation, there is still no clarity about the physical interpretation of the quantum mechanical "probability wave function".

This manuscript describes a third unknown interpretation of the quantum mechanical "probability wave function". A fundamental physical interpretation that will change the generally accepted Copenhagen Interpretation. The manuscript will try to answer the fundamental question: Does an alternative theory exist in which the existence of this particular photo, of the quantum mechanical probability wave within the hydrogen atom, could be explained? And can the famous photo of the hydrogen atom be explained in a logical and a simple way?

To answer this, we have to go back in time about 300 years ago, to the moment when Isaac Newton discovered one of the many secrets of light. And we return to the fundamental question: "Is it possible to build a matter out of Light (confined electromagnetic waves)?" To answer this question, we first have to answer the question how we have come to this world of probability waves and elementary particles and quantum physics.

Louis de Broglie described in 1924 in his PhD thesis, the wave properties of matter. Erwin Schrödinger published in 1926 the well-known Schrödinger wave equation with the characteristic spherical and elliptical wave solutions. To describe these material waves, an immediate and first explanation was: "There is only one possibility. These are 'electromagnetic waves'. De Broglie Waves are 'light waves'. These were the only waves, known in the world of Physics that could travel through vacuum. 
But there was a fundamental problem. These material waves, discovered by Louis de Broglie and mathematically described by Erwin Schrödinger could never be solutions of the wellknown 4 linear equations of James Clerk Maxwell. The material waves were spherical and elliptical solutions of confinement and, thus, cannot be solutions of the linear maxwell's equations according to which, superposition is possible but electromagnetic interactions and electromagnetic confinement can only be described by a set of non-linear differential equations. To solve this problem, a new set of 3 non-linear Electromagnetic Equations (Equation 5 in this manuscript) has been developed and it replaces the well-known 4 linear Maxwell Equations and the possible solutions of equation (5) are also spherical and elliptical solutions of confinement. Table 1 in this manuscript presents a number of electromagnetic spherical confinements as solutions of equation 5 a.

A second problem was the model of the atom of Bohr that could not hold. How an electron could be everywhere in a kind of a spherical shell surrounding the nucleus, and be a particle at the same time?

Bohr found a compromise to declare the 'De Broglie' waves to be probability waves. Two problems were solved. Bohr's planetary model could hold. And there was a name for the unknown material waves, discovered by Louis de Broglie and mathematically described by Erwin Schrödinger. The theory of 'probability waves, elementary particles and quantum physics' had been created.

To create matter out of light, we have to go back to the Equations of James Clerk Maxwell. The only possibility to declare the probability waves, discovered by Louis de Broglie, to be electromagnetic waves, to be light, and to be the building element of our universe, is to find evidence that Maxwell's Equations are not complete and it is possible to confine light (electromagnetic waves) in spherical and elliptical shapes. Therefore, a new equation needs to be found. In this article, it has been claimed that the missing equation is the equation (5). And as the Theory of General Relativity has been built on Einstein-Maxwell Equation, a new equation has also to be found to describe in a generalized way the interaction between light and matter. This missing equation is the equation (5-a) described in this paper.

\section{Introduction [NB. All calculations have been made in Mathematica 11.0]}

To create a matter out of light, we have to go back to James Clerk Maxwell's equations. The only possibility to declare the probability waves, discovered by Louis de Broglie, to be electromagnetic waves, to be light, and to be a building element of our universe, is to find evidence that Maxwell's equations are not complete and that it is possible to confine light (electromagnetic waves) in spherical and elliptical shapes. A new equation has to be found. In this article, it has been claimed that this missing equation is the equation (5). Because the theory of general relativity has been built on the Einstein-Maxwell's equation, a new equation 
has to be found that describes the interaction between light and matter in a generalized way. This missing equation is equation (5-a).

The first step to be taken is to find evidence that 'de Broglie Waves' (material waves, quantum mechanical probability waves) are 'confined single harmonic electromagnetic waves' is to find a new electromagnetic equation that will replace the well-known 4 Maxwell Equations. James Clerk Maxwell published in 1865 in "A Dynamical Theory of the Electromagnetic Field" the 4 well-known Maxwell's equations. To find an equation that describes in an extended way the Electromagnetic Field, we have to go back to the time before the Maxwell's equations. The Maxwell's equations have been called the "second great unification in physics" after the "first unification in physics, realized by Isaac Newton. And that is the time we have to go back to, to discover a new equation, that unifies the electric and the magnetic field in a generalized way.

The second step to be taken, is that confined electromagnetic waves have to be presented in a complex way comparable with the quantum mechanical probability function $\psi$ and the complex conjugate function $\psi^{*}$ in which the quantum mechanical probability density $\psi \psi^{*}$ will be replaced by the electromagnetic mass density. This second step has been taken in an earlier publication (29) in which a complex notation for the electromagnetic field has been introduced in the equations (13), (14) and (15) on page 202, which has been published in 1995 in Physics Essays in a continuous model of matter (DOI: 10.13140/RG.2.2.25149.77281). (Ref. 29)

In Ref (29) in equation (13) the vector function $\vec{\phi}=\vec{B}+i \vec{E} / c$ and the complex conjugate vector function $\vec{\phi}=\vec{B}-\mathrm{i} \overrightarrow{\mathrm{E}} / \mathrm{c}$ have been introduced in which $\overrightarrow{\mathrm{B}}$ is the magnetic induction vector, $\overrightarrow{\mathrm{E}}$ the electric field intensity vector and c the speed of light. Equation (14) represents the dot product $\vec{\phi} \square \vec{\phi}^{*}=\mathrm{B}^{2}+\mathrm{E}^{2} / \mathrm{c}^{2} \square \mathrm{w}$ which is proportional to the electromagnetic energy density $\mathrm{w}$ and according to Einstein's equation $W=\mathrm{mc}^{2}$ proportional to the electromagnetic mass density $\mathrm{Q}$. The cross product $\vec{\phi} \otimes \vec{\phi}^{*}$ in equation (15) is proportional to the momentum of the confined single harmonic electromagnetic radiation. The third step that has to be taken is to provide evidence that the well-known 'de Broglie' waves are solutions of the 'Electromagnetic Continuity Equation' for 'Confined Single Harmonic Electromagnetic Waves' with one single harmonic Frequency $\omega_{0}$. And that the Quantum Mechanical Schrödinger Wave Equation is a complex notation presented in Ref. (29) in equation (55) on page 208 in which the complex notation for the Electromagnetic Continuity Equation has been presented which turns out to be identical to the well-known Quantum Mechanical Schrödinger Wave Equation. This has been published in 1995 in Physics Essays in A Continuous model of Matter (DOI: 10.13140/RG.2.2.25149.77281). (Ref. 29)

A fourth step is to provide evidence that the well-known 'de Broglie' waves at relativistic velocities are solutions of the 'electromagnetic continuity equation' for 'confined single 
harmonic electromagnetic waves' with one single harmonic frequency $\omega_{0}$. And that the quantum mechanical Dirac equation is a complex notation for the electromagnetic continuity equation for confined single harmonic electromagnetic waves. This evidence has been presented in Ref. (29) in equation (102) on page 213 in which the complex notation for the electromagnetic continuity equation has been presented which turns out to be identical to the well-known quantum mechanical dirac equation at relativistic velocities. This has been published in 1995 in Physics Essays in A continuous model of matter (DOI: 10.13140/RG.2.2.25149.77281). (Ref. 29)

The famous eclipse expedition of 1919 to Sobral, Brazil, and the island of Principe, in the Gulf of Guinea, led by Dyson, Eddington and Davidson was a turning point in the acceptance of the general theory of relativity developed by Albert Einstein in 1915. The correctness of this theory has been proven since then in many experiments. The success of his predictions made Einstein famous. Albert Einstein published his famous 'Einstein Field Equations' (Einstein-Maxwell equations) in 1915, which were the mathematical foundation on which the Theory of General Relativity has been built. Einstein built his theory together with Hermann Minkowski using the complex 4-dimensional Minkowski Space and Hendrik Antoon Lorentz using the Electromagnetic Lorentz Transformations, developed by Hendrik Antoon Lorentz .

In this manuscript, the starting point is almost 300 years ago. Long before James Maxwell published his Maxwell equations, before Albert Einstein published the Theory of General Relativity and far before the time Niels Bohr introduced the probability waves, discovered by Erwin Schrödinger which were illogical grounds on which Quantum Physics has been built.

The starting point is the moment just after Isaac Newton had published the breaking of light into the colors of the rainbow through a prism in The Lectiones Opticae" in 1728. Newton just had discovered the beauty of light when light had revealed one of its many secrets. And he could only express: "Light is beautiful".

When we return back to the world of light, we simply have to ask one question. How is it possible that the light in our world does exist? And we simply follow Newton's third law of equilibrium published in the "Principia Mathematica Philisophiae Naturalis" in 1686.

Another fundamental question has to be answered: What are the fundamental boundaries required for a stable electromagnetic field configuration in which light can exist? And instead of taking the 4 well-known electromagnetic low frequency equations and put them together and find exactly the same results as James Maxwell has found, a different path has been chosen. There is only one boundary condition. "The electromagnetic field has to be in a perfect equilibrium (balance) with itself and its surroundings." And when an electromagnetic field interacts with a gravitational field, exactly the same boundary condition is required. That is the single and only requirement. From this single requirement follows one single equation. Equation (5) (gravity excluded) and equation (5-a) (gravity included) in this manuscript.

All the solutions for electromagnetic configurations that are solutions of the Maxwell's equations are also solutions of equation (5). But because of the special mathematical structure 
of equation (5) and (5-a), other electromagnetic field configurations are possible which are nonlinear solutions of $(5)$ and $(5-a)$. And this will have an impact on our understanding of the universe, on our understanding of light, because we cannot understand the universe until we understand light.

John Archibald Wheeler ${ }^{(1)}$ introduced in 1953 the concept of GEONS (Gravitational ElectrOmagnetic eNtities) in which electromagnetic radiation has been confined by its own gravitational field. To calculate the dimensions of these gravitational-electromagnetic confinements J. Wheeler based his calculations on the Einstein-Maxwell equations, the mathematical ground on which the Theory of General Relativity has been built and found electromagnetic-gravitational confinements with a diameter of several light years and a lifetime of several milliseconds. The results were very disappointing because an elementary particle with a diameter of several light years and a lifetime of a few milliseconds can hardly be considered as an elementary particle.

In the present theory, the electromagnetic-gravitational interaction has been grounded on Newton's third law. It has been grounded on the stability of electromagnetic-gravitational fields in a perfect equilibrium with itself and its surroundings. In table 1, electromagnetic-gravitational confinements have been presented for any harmonic (sinusoidal) frequency with an infinite lifetime and diameters varying from $<10^{-40}$ and $>10^{+40}[\mathrm{~m}]$ based on equation $5-a$ in a spherical coordinate system.

Because this fundamental approach in electromagnetic field configurations is different than the classical approach by J. Maxwell and A. Einstein, a different result is expected. Instead of electromagnetic-gravitational confinements with a diameter of several light years and a lifetime of several milliseconds, presented by J. Wheeler in 1953, electromagnetic-gravitational confinements have been presented in Table 1 with diameters varying from $<10^{-40}[\mathrm{~m}]$ and $>10^{+40}[\mathrm{~m}]$ and an infinite lifetime for any harmonic frequency based on equation (5-a).

\section{Newton's Third law generalized for Electromagnetic Radiation}

In a way comparable to the way that GEONS (Gravitational ElectrO-magnetic eNtities) are described by J. Wheeler in General Relativity by the Gravitational-Electromagnetic Equilibrium Equation (1,7,8,13,35,38) (the Einstein-Maxwell Equations), Electromagnetic Confinements are described by the Dynamic Equilibrium Equation (5).

Newton's Third law has been generalized in all layers of Physics. There is no reason not to generalize Newton's third law also within electromagnetic fields. To generalize Newton's third law within Electromagnetic Field Configurations, the Divergence has been taken of the Stress Energy Tensor (3), which results in Equation (4) to calculate the force densities $f$ within the Electromagnetic Field Configuration.

The Energy Momentum Tensor ${ }^{(9)}$ equals: 


$$
T^{a b}=\frac{1}{\mu_{0}}\left[F_{a c} F^{c b}+\frac{1}{4} \delta_{a b} F_{c d} F^{c d}\right]
$$

In which $F_{a b}$ are the elements of the Maxwell Tensor defined by:

$$
F_{a b}=\partial_{b} \varphi_{a}-\partial_{a} \varphi_{b}
$$

The four-vector potential $\varphi_{a}$ is defined by: $\varphi_{\mathrm{a}}=\{\mathrm{i} \varphi / \mathrm{c}, \overrightarrow{\mathrm{A}}\}$ where $\varphi$ is the electric scalar potential, c the speed of light in vacuum and $\vec{A}$ is the magnetic vector potential ${ }^{(1,2,3)}$. Substituting (2) in (1) results in the Stress Energy Tensor ${ }^{(1,16,29)}$. :

$$
T^{a b}=\left[\begin{array}{rrrr}
w & -\frac{i}{c} S_{x} & -\frac{i}{c} S_{y} & -\frac{i}{c} S_{z} \\
-\frac{i}{c} S_{x} & \varepsilon_{0}\left(E_{x}\right)^{2}+\mu_{0}\left(H_{x}\right)^{2}-w & \varepsilon_{0} E_{x} E_{y}+\mu_{0} H_{x} H_{y} & \varepsilon_{0} E_{x} E_{z}+\mu_{0} H_{x} H_{z} \\
-\frac{i}{c} S_{y} & \varepsilon_{0} E_{y} E_{x}+\mu_{0} H_{y} H_{x} & \varepsilon_{0}\left(E_{y}\right)^{2}+\mu_{0}\left(H_{y}\right)^{2}-w & \varepsilon_{0} E_{y} E_{z}+\mu_{0} H_{y} H_{z} \\
-\frac{i}{c} S_{z} & \varepsilon_{0} E_{z} E_{x}+\mu_{0} H_{z} H_{x} & \varepsilon_{0} E_{z} E_{x y}+\mu_{0} H_{z} H_{y} & \varepsilon_{0}\left(E_{z}\right)^{2}+\mu_{0}\left(H_{z}\right)^{2}-w
\end{array}\right]
$$

In the absence of any Gravity, the force density $f^{a}$ in the 3 directions of the 3 coordinates of the chosen 3-coordinate system follows from the (4-dimensional) Divergence of the (4dimensional) Stress Energy Tensor ${ }^{(8,9,38)}$ (3).

The Divergence of a Vector equals a Scalar. The Divergence of a Tensor equals a Vector. The 4dimensional Divergence of the 4-dimensional Stress Energy Tensor (4) equals the $\underline{4-d i m e n s i o n a l}$ Force-density Vector. The first 3 terms of the 4-dimensional Force-density vector equal the force densities in the corresponding 3 dimensions of the chosen Coordinate System. The $4^{\text {th }}$ component equals the imaginary component of the time derivative of the energy density divided by the speed of light.

To calculate the equilibrium conditions in the Electromagnetic Field Configuration, the first 3 terms of the 4-dimensional Force-density vector are being used.

By rearranging the first 3 terms of the (4-dimensional Divergence) of the (4-dimensional) Stress Energy Tensor (4) an equation for the 3-dimensional force density $f^{a}$ within the Electromagnetic Field Configuration has been created. This Equation (4) represents the $\underline{3-}$ 
dimensional force density $f^{a}$ in a coordinate-free vector equation in the absence of any Gravity:

$$
-\frac{1}{c^{2}} \frac{\partial(\vec{E} \times \vec{H})}{\partial t}+\varepsilon_{0} \vec{E}(\nabla \vec{E})-\varepsilon_{0} \vec{E} \times(\nabla \times \vec{E})+\mu_{0} \vec{H}(\nabla \square \overrightarrow{\mathrm{H}})-\mu_{0} \overrightarrow{\mathrm{H}} \times(\nabla \times \vec{H})=\vec{f}
$$

According to Newton's third law, the force density $\vec{f}$ has to equal zero for any physical possible electromagnetic configuration (free radiation or confinement). According to Newton's third law, it is the only required and sufficient boundary condition that the force densities in the 3 dimensions of the chosen coordinate system equal zero at any space at any time.

An electromagnetic field which is in a perfect equilibrium with itself and its surrounding at any space and time, fulfills the necessary requirements of Newton's third law for the physical possibility of the existence of this field. Under that condition Equation (4) transforms into the Dynamic Equilibrium Equation (5), which expresses the force density equals the vector zero of an electromagnetic field on itself and its surroundings in a perfect equilibrium. The part of the Dynamic Equilibrium Equation ${ }^{(7,8,9.20)}$ (DEE) (5), representing the equilibrium for the force

$$
-\frac{1}{c^{2}} \frac{\partial(\vec{E} \times \vec{H})}{\partial t}+\varepsilon_{0} \overrightarrow{\mathrm{E}}(\nabla \overrightarrow{\mathrm{E}})-\varepsilon_{0} \overrightarrow{\mathrm{E}} \times(\nabla \times \overrightarrow{\mathrm{E}})+\mu_{0} \vec{H}(\nabla \vec{H})-\mu_{0} \vec{H} \times(\nabla \times \vec{H})=\overrightarrow{0}
$$

densities in the 3 directions of the chosen coordinate system equals: The resulting REM equation equals:

Substituting the well-known 4 Maxwell Equations in vacuum into (5) results in:

$$
-\varepsilon_{0} \mu_{0} \frac{\partial(\vec{E} \times \vec{H})}{\partial t}+\varepsilon_{0} \overrightarrow{\mathrm{E}}(\nabla \overrightarrow{\mathrm{E}})-\varepsilon_{0} \mu_{0} \overrightarrow{\mathrm{E}} \times\left(-\frac{\partial(\vec{H})}{\partial t}\right)+\mu_{0} \vec{H}(\nabla \vec{H})+\mu_{0} \vec{H} \times\left(\varepsilon_{0} \frac{\partial(\vec{E})}{\partial t}\right)=\overrightarrow{0}
$$

According to Maxwell's equations, the electromagnetic field is divergence free. And (5.1) results in:

$$
-\varepsilon_{0} \mu_{0} \frac{\partial(\vec{E} \times \vec{H})}{\partial t}-\varepsilon_{0} \mu_{0} \overrightarrow{\mathrm{E}} \times\left(-\frac{\partial(\vec{H})}{\partial t}\right)+\mu_{0} \vec{H} \times\left(\varepsilon_{0} \frac{\partial(\vec{E})}{\partial t}\right)=\overrightarrow{0}
$$

Equation 5.2 is a well-known vector identity and the left side also equals zero. Equation (5) is a much more powerful Equation than the four well-known 4 Maxwell Equations. Equation (5) includes all the $\underline{4}$ Maxwell Equations in vacuum. This implies that every solution of the $\underline{4}$ Maxwell Equations in vacuum is also a solution of Equation (5).

But in contradiction to the solutions for the 4 Maxwell Equations there is no requirement that for Equation (5) the electromagnetic field has to be Divergence-Free. And in physics this is very 
often a fact. A laser beam with a Gaussian Intensity Division is never Divergence-free. But it is a perfect solution of Equation (5). Equation (5) offers unique possibilities for Electromagnetic Interactions and for Electromagnetic Confinements. And that powerful fact makes Equation (5) unique and will change the possibilities in the Electromagnetic Field Configurations dramatically. The Relativistic Gravitational Electro Magnetic (RGEM) equation within a gravitational field $\vec{g}$ equals to:

$$
\begin{aligned}
& -\frac{1}{c^{2}} \frac{\partial(\vec{E} \times \vec{H})}{\partial t}+\varepsilon_{0} \overrightarrow{\mathrm{E}}(\nabla \overrightarrow{\mathrm{E}})-\varepsilon_{0} \overrightarrow{\mathrm{E}} \times(\nabla \times \overrightarrow{\mathrm{E}})+\mu_{0} \vec{H}(\nabla \overrightarrow{\vec{H}})-\mu_{0} \vec{H} \times(\nabla \times \vec{H}) \\
& -\frac{1}{2} \varepsilon_{0}^{2} \mu_{0}(\overrightarrow{\mathrm{E}} \overrightarrow{\mathrm{E}}) \overrightarrow{\mathrm{g}}-\frac{1}{2} \varepsilon_{0} \mu_{0}^{2}(\vec{H} \vec{H}) \overrightarrow{\mathrm{g}}=\overrightarrow{0}
\end{aligned}
$$

\section{1 EM Radiation within a Cartesian Coordinate System in the absence of gravity}

The required Electromagnetic Field Configuration for a perfect Equilibrium in Space and Time follows from the dynamic equilibrium equation (5) and equals in Cartesian Coordinates

$$
\left(\begin{array}{l}
e_{x} \\
e_{y} \\
e_{z}
\end{array}\right)=\left(f(x, y) g\left(t-\left(\frac{\mathrm{K}_{1}}{z}+1\right) z \sqrt{\varepsilon_{0} \mu_{0}}\right)\right)
$$

$\{x, y, z, t\}$ for the Electric Field Components e $(x, y, z, t)$ :

The required Electromagnetic Field Configuration for a perfect Equilibrium in Space and Time follows from the dynamic equilibrium equation (5) and equals in Cartesian Coordinates $\{x, y, z, t\}$ for the Magnetic Field Components $\mathrm{m}(x, y, z, t)$ : 


$$
\left(\begin{array}{l}
m_{x} \\
m_{y} \\
m_{z}
\end{array}\right)=\sqrt{\frac{\varepsilon_{0}}{\mu_{0}}}\left(f(x, y) g\left(t-\left(\frac{\mathrm{K}_{1}}{z}+1\right) z \sqrt{\varepsilon_{0} \mu_{0}}\right)\right)
$$

In which $K_{1}$ is an arbitrary constant. For the divergence-free function $f(x, y)=1$, the solutions (6) and (7) also are the solutions for the known Maxwell Equations. For the non-divergence-free functions $f(x, y)$, the solutions (6) and (7) are not solutions for the Maxwell Equations, which requires divergence-free electromagnetic waves, propagating with the speed of light $c=1 / \sqrt{\varepsilon_{0} \mu_{0}}$, in the absence of any matter. But they are solutions of the Dynamic Equilibrium Equation (5) and clearly do exist in physics. Comparable with the projection of a slide with a beamer on a flat screen in the z-direction. In which the slide has an arbitrary intensity division $f(x, y)$. The information $f(x, y)$ on the slide propagates with the speed of light $c=1 / \sqrt{\varepsilon_{0} \mu_{0}}$ towards the screen in the $z$-direction in this example.

\section{2 EM Radiation within a Cartesian Coordinate System under the influence of a Longitudinal Gravitational Field $g$}

The required Electromagnetic Field Configuration for a perfect Equilibrium in Space and Time for a Longitudinal Gravitational Field (The Light propagates in the same z-direction as the zdirection of the Gravitational Field) follows from the Dynamic Equilibrium Equation (5-a) and equals in Cartesian Coordinates $\{x, y, z, t\}$ for a gravitational field " $\mathrm{g}$ " for the Electric Field Components e $(x, y, z, t)$ :

$$
\left(\begin{array}{l}
e_{x} \\
e_{y} \\
e_{z}
\end{array}\right)=\left(\begin{array}{cc}
\mathrm{e}^{-\frac{1}{2} \mathrm{~g} \varepsilon_{0} \mu_{0} \mathrm{z}} & f(x, y) g\left(t-z \sqrt{\varepsilon_{0} \mu_{0}}\right) \\
0 \\
0
\end{array}\right)
$$

The required Electromagnetic Field Configuration for a perfect Equilibrium in Space and Time for a Longitudinal Gravitational Field (The Light propagates in the same z-direction as the zdirection of the Gravitational Field ) follows from the Dynamic Equilibrium Equation (5-a) and 
equals in Cartesian Coordinates $\{x, y, z, t\}$ for a gravitational field " $\mathrm{g}$ " for the Magnetic Field Components $\mathrm{m}(x, y, z, t)$ :

$$
\left(\begin{array}{l}
m_{x} \\
m_{y} \\
m_{z}
\end{array}\right)=\sqrt{\frac{\varepsilon_{0}}{\mu_{0}}}\left(\begin{array}{c}
\mathrm{e}^{-\frac{1}{2} \mathrm{~g} \varepsilon_{0} \mu_{0} \mathrm{z}} \\
f(x, y) g\left(t-z \sqrt{\varepsilon_{0} \mu_{0}}\right) \\
0
\end{array}\right)
$$

Equation (6-a) and (7-a) are solutions of (5-a) under the influence of a Longitudinal Gravitational field with field intensity " $g$ " that acts along the z-direction while the electromagnetic wave is also propagating in the z-direction. The electromagnetic wave is propagating with the unaltered speed of light $c=1 / \sqrt{\varepsilon_{0} \mu_{0}}$, independently of the strength $\mathrm{g}$ of the gravitational field in the $\mathrm{z}$ direction. However, the amplitude of the electromagnetic wave becomes dependently of the gravitational intensity " $\mathrm{g}$ " and the distance " $\mathrm{z}$ " and changes along the $\mathrm{z}$-axis with the electromagnetic-gravitational interaction term $\mathrm{e}^{-\frac{1}{2} \mathrm{~g} \varepsilon_{0} \mu_{0} \mathrm{z}}$.

In this example is chosen for e.g. a laser beam positioned vertically on the ground on earth, shining vertically against the gravitational field " $\mathrm{g}$ " of the earth. Because the laser beam presents electromagnetic energy, the beam has electromagnetic mass. The potential energy of the electromagnetic mass is increasing while the laser light is propagating upwards, against the direction of the gravitational field. Because of the law of conservation of Energy, the electromagnetic energy is decreasing over a distance " $z$ " proportional with the same amount $\mathrm{e}^{-\mathrm{g} \varepsilon_{0} \mu_{0} \mathrm{z}}$ as the potential energy of the electromagnetic mass is increasing.

\section{Electromagnetic Radiation within a Spherical Coordinate System}

The Spherical Coordinate System $\{r, \theta, \varphi, t\}$ is parameterized by the radius $r$ of the Sphere, the polar angle $\theta$ and the azimuthal angle $\varphi$ and the time t.

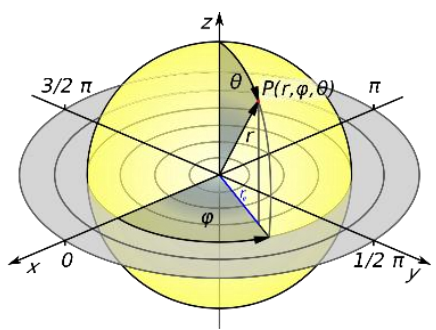


The required Electromagnetic Field Configuration for a perfect Equilibrium in Space and Time follows from equation (5) and equals in Spherical Coordinates $\{r, \theta, \varphi, t\}$ for the Electric Field Components e $(\theta, r, \varphi, t)$ :

$$
\left(\begin{array}{l}
e_{r} \\
e_{\theta} \\
e_{\varphi}
\end{array}\right)=\left(\begin{array}{c}
0 \\
\frac{1}{r} f(\theta, \varphi) g\left(t-\left(\frac{\mathrm{K}_{1}}{r}+1\right) r \sqrt{\varepsilon_{0} \mu_{0}}\right) \\
0
\end{array}\right)
$$

The required Electromagnetic Field Configuration for a perfect Equilibrium in Space and Time follows from equation (4) and equals in Spherical Coordinates $\{r, \theta, \varphi, t\}$ for the Magnetic Field Components $\mathrm{m}(\theta, r, \varphi, t)$ :

$$
\left(\begin{array}{l}
m_{r} \\
m_{\theta} \\
m_{\varphi}
\end{array}\right)=\sqrt{\frac{\varepsilon_{0}}{\mu_{o}}}\left(\begin{array}{c}
0 \\
0 \\
\frac{1}{r} f(\theta, \varphi) g\left(t-\left(\frac{\mathrm{K}_{1}}{r}+1\right) r \sqrt{\varepsilon_{0} \mu_{0}}\right)
\end{array}\right)
$$

For the divergence-free function $f(\theta, \varphi)=1$, the solutions (8) and (9) also are the solutions for the known Maxwell Equations. For the non-divergence-free functions $f(\theta, \varphi)$, the solutions (8) and (9) are no solutions for the Maxwell Equations, which require divergence-free electromagnetic waves in the absence of any matter. ${ }^{(29,35,36,37,38)}$. They are, however, solutions of the $\underline{D E E}(5)$ and clearly they do exist in physics. Like the radiation of an inhomogeneous point light source like a LED.

\section{Confined Electromagnetic Radiation within a Spherical Coordinate System through Electromagnetic-Gravitational Interaction.}

In physics, it has been assumed generally that the speed of light $c=1 / \sqrt{\varepsilon_{0} \mu_{0}}$ is a physical constant. In this paragraph, the possibilities will be discussed of a variable speed of light ${ }^{(10,11,12)}$, which can vary from zero up to values higher than c. The only requirement for the existence of an Electromagnetic Field Configuration will be the requirement of a perfect equilibrium in space- 
time for the chosen electromagnetic field configuration ${ }^{(13,14,15)}$. This single unique requirement will always be a solution of the DEE (5).

The required Electromagnetic Field Configuration for a perfect Equilibrium in Space and Time ${ }_{(16,17,18,30,31,39}$ in respectively the $: \theta$-direction $\left(f_{\theta}=0\right)$ and the $\varphi$-direction: $(f \varphi=0)$ follows from equation (5). In Spherical Coordinates $\{r, \theta, \varphi, t\}$ the solution for the $\underline{D E E}(5)$ for the Electric Field Components e $(\theta, \mathrm{r}, \varphi, \mathrm{t})$ equals to:

$$
\left(\begin{array}{c}
e_{r} \\
e_{\theta} \\
e_{\varphi}
\end{array}\right)=\left(\begin{array}{c}
0 \\
f(r) g(\theta) h(\varphi) \operatorname{Sin}(\omega t) \\
-f(r) g(\theta) h(\varphi) \operatorname{Cos}(\omega t)
\end{array}\right)
$$

In Spherical Coordinates $\{r, \theta, \varphi, t\}$ the solution for the DEE (5) for the Magnetic Field Components $\mathrm{m}(\theta, \mathrm{r}, \varphi, \mathrm{t})$ in respectively the: $\theta$-direction $\left(f_{\theta}=0\right)$ and the $\varphi$-direction : $(f \varphi=0)$ for the magnetic field components follows from equation $(5)^{(29,30,31)}$ and equals to:

$$
\left(\begin{array}{l}
m_{r} \\
m_{\theta} \\
m_{\varphi}
\end{array}\right)=\left(\begin{array}{c}
0 \\
f(r) g(\theta) h(\varphi) \operatorname{Cos}(\omega t) \\
f(r) g(\theta) h(\varphi) \operatorname{Sin}(\omega t)
\end{array}\right)
$$

Equation (4) gives the 3-dimensional force density $f^{a}$ of an Electro-Magnetic Field Configuration ${ }^{(19,20,21)}$ in a coordinate free vector equation. It follows from equation (4) that the radiation pressure in radial direction does not

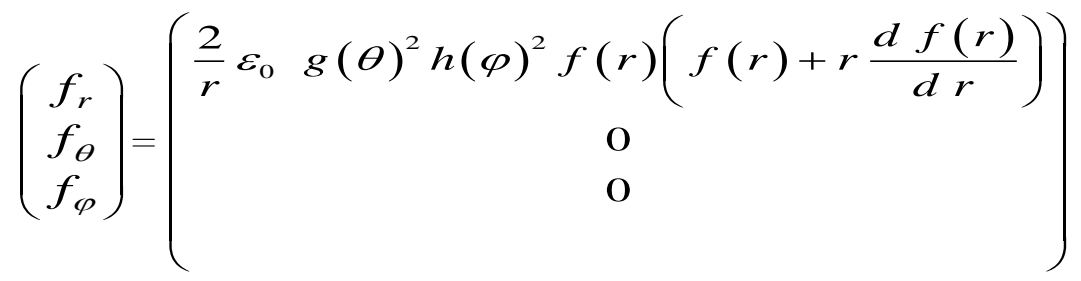

counterbalance and does not equal zero. 
The energy density $\mathrm{w}_{\mathrm{m}}$ of the Electromagnetic Configuration is essential for the calculation of the inward bounded gravitational pressure. The electromagnetic field configuration (10) and (11) for the functions $g(\theta)=1$ and $h(\varphi)=1$, results into the electromagnetic energy density $\mathrm{W}_{\mathrm{em}}$ :

$$
\begin{aligned}
& \left(\begin{array}{l}
e_{r} \\
e_{\theta} \\
e_{\varphi}
\end{array}\right)=\left(\begin{array}{c}
0 \\
\mathrm{f}(\mathrm{r}) \operatorname{Sin}(\omega \mathrm{t}) \\
-\mathrm{f}(\mathrm{r}) \operatorname{Cos}(\omega \mathrm{t})
\end{array}\right) \quad\left(\begin{array}{l}
m_{r} \\
m_{\theta} \\
m_{\varphi}
\end{array}\right)=\left(\begin{array}{c}
0 \\
\mathrm{f}(\mathrm{r}) \operatorname{Cos}(\omega \mathrm{t}) \\
\mathrm{f}(\mathrm{r}) \operatorname{Sin}(\omega \mathrm{t})
\end{array}\right) \\
& \mathrm{w}_{\mathrm{em}}=\left(\frac{\mu_{0}}{2}(\overrightarrow{\mathrm{m}} \square \overrightarrow{\mathrm{m}})+\frac{\varepsilon_{0}}{2}(\overrightarrow{\mathrm{e}} \square \overrightarrow{\mathrm{e}})\right)=\varepsilon_{0} \mathrm{f}(r)^{2}
\end{aligned}
$$

According to Einstein's mass-energy equivalent $\mathrm{W}=\mathrm{mc}^{2}$, the specific electromagnetic mass ${ }^{(22,23,24,29)}$ density $\rho_{\text {em }}$ equals to:

$$
\rho_{\mathrm{em}}=\frac{1}{\mathrm{c}^{2}}\left(\frac{\mu_{0}}{2}(\overrightarrow{\mathrm{m}} \square \overrightarrow{\mathrm{m}})+\frac{\varepsilon_{0}}{2}(\overrightarrow{\mathrm{e}} \square \overrightarrow{\mathrm{e}})\right)=\varepsilon_{0}{ }^{2} \mu_{0} \mathrm{f}(\mathrm{r})^{2}
$$

The total electromagnetic mass ${ }^{(25,6,27,29,38)} . M_{e m}$ within a sphere with radius $R$ equals $t$ :

$$
\mathrm{M}_{e m}=4 \pi \varepsilon_{0}{ }^{2} \mu_{0} \int_{0}^{\mathrm{R}} \mathrm{r}^{2} \mathrm{f}(\mathrm{r})^{2} \mathrm{dr}
$$

At a distance $r$ from the center of the sphere, the total electromagnetic mass $M_{e m}$ within the sphere ${ }^{(25,26,27,30)}$ causes, according to Newton's Shell Theorem ${ }^{(28,29,30)}$, a gravitational field strength $g_{\text {em }}:$

$$
\mathrm{g}_{\mathrm{em}}=\frac{4}{\mathrm{r}^{2}} \pi \varepsilon_{0}^{2} \mu_{o} \mathrm{G}_{1} \int_{0}^{\mathrm{R}} \mathrm{r}^{2} \mathrm{f}(\mathrm{r})^{2} \mathrm{dr}
$$


In which $G_{1}$ is the gravitational constant and equals to $G_{1}=6.6740810^{-11}\left[\mathrm{~m}^{3} \mathrm{~kg}^{-1} \mathrm{~s}^{-2}\right]$. The gravitational inwards bounded radiation pressure ${ }^{(29,31,32,33)}$ follows from (16):

$$
\mathrm{f}_{G R A V}=\rho_{\mathrm{em}} \mathrm{g}_{\mathrm{em}}=\frac{4}{\mathrm{r}^{2}} \pi \varepsilon_{0}^{4} \mu_{0}^{2} \mathrm{G}_{1} \mathrm{f}(r)^{2} \int_{0}^{\mathrm{R}} \mathrm{r}^{2} \mathrm{f}(\mathrm{r})^{2} \mathrm{dr}
$$

When there is a perfect equilibrium between the outwards bounded electromagnetic

radiation pressure $f_{R A D}$ and the inward bounded gravitational pressure ${ }^{(29,34,35,38,39)}$. $f_{G R A V}$ we find from (12) and (17) the radius of the boundary sphere of the enclosed radiation $R_{B O U N D A R Y}$. for the functions $\mathrm{g}(\theta)=1$ and $\mathrm{h}(\varphi)=1$,

$$
\mathrm{f}_{\text {RAD }}=\frac{2}{\mathrm{r}} \varepsilon_{0} \mathrm{f}(\mathrm{r})\left(\mathrm{f}(\mathrm{r})+\mathrm{r} \frac{\mathrm{d} \mathrm{f}(\mathrm{r})}{\mathrm{dr}}\right)=\frac{4}{\mathrm{r}^{2}} \pi \varepsilon_{0}^{4} \mu_{0}^{2} \mathrm{G}_{1} \mathrm{f}(\mathrm{r})^{2} \int^{\text {R-BOUMNDAAY }} \mathrm{r}^{2} \mathrm{f}(\mathrm{r})^{2} \mathrm{dr}=\mathrm{f}_{\text {GRAV }}
$$

In the following example, we choose for the function $f(r)$

$$
f(r)=K_{1} r^{n}
$$

Substituting (19) in (18) results in the equation for $R_{\text {BOUNDARY }}$ :

$\frac{8.019056 \times 10^{-94} \mathrm{~K}^{6} \mathrm{R}_{\text {BOUNDARY }}^{1+6 n}}{3+2 \mathrm{n}}=1.7708376 \times 10^{-11} \mathrm{~K}^{2}(1+\mathrm{n}) \mathrm{R}_{\text {BOUNDARY }}^{-1+2 n}$

\section{The fundamental conflict between Causality and Probability}

The beginning of the conflict between Causality and Probability in Physics started at the historic invitation only "Conseil Solvay" in 1911. Since that conference Albert Einstein has always defended the fundamental concept of Causality and the logical grounds for Causality and Effect while Niels Bohr has always defended the fundamental concept of Probability in which there is no relationship between Causality and Effect. The most fundamental and famous conference was the October 1927 "Fifth Solvay International Conference on Electrons and Photons" where the newly formulated Quantum Theory, based on Probability, had been 
accepted. Since then Quantum Physics has grown in power and has always been grounded on the material waves, for the first time mathematically described by Erwin Schrödinger and designated to be probability waves.

In Ref. (29) page 206 the "law of continuity" for electromagnetic radiation has been presented in equation (42), By presenting the electromagnetic field in a complex configuration in equation (48) on page 207, the electromagnetic continuity equation (42) has been presented as the quantum mechanical Schrödinger wave equation (53) on page 207. However the function $\psi$ in (53) does not represent the quantum mechanical probability function but represents the confined electromagnetic field with harmonic frequency $\omega_{0}$ in which the magnetic part has been presented as the real function $\vec{B} / \mu$ and the electric part has been presented as the imaginary function $i \overrightarrow{\mathrm{E}} / \mathrm{c}$. With "i" the imaginary number $\sqrt{-1}$. However, as well as the electric part as the magnetic part of the confined electromagnetic field are both real.

In Ref. (29) on page 208 the "law of continuity" for electromagnetic radiation has been presented in equation (57). The vector function $\vec{\phi}$ represents the confined electromagnetic field with harmonic frequency $\omega_{0}$ in which the magnetic part has been presented as the real vector function $\vec{B} / \mu$ and the electric part has been presented as the imaginary vector function $i \overrightarrow{\mathrm{E}} / \mathrm{c}$. By substituting the complex vector function $\vec{\phi}$ in (57) and using the relativistic Lorentz transformations, the quantum mechanical relativistic Dirac equation has been derived in equation (102) on page 213. This has been published in 1995 in Physics Essays in A Continuous model of Matter (DOI: 10.13140/RG.2.2.25149.77281). (ref. 29).

This fundamental conflict is still going on and in this article mathematical results are presented in Table 1 in which the famous De Broglie waves, the material waves, designated as probability waves are electromagnetic waves with one harmonic frequency $\omega$, confined by electromagnetic-gravitational interaction. Electromagnetic waves fully grounded on Causality and Effect.

Table 1 below presents several values for the calculated Equilibrium Radius $R_{\text {BOUNDARY }}(20)$ at different values $K_{1}$ and $n$ in Equation (18), (19) and (20) for the harmonic ElectromagneticGravitationally confined Electromagnetic waves with frequency $\omega_{0}$ :

\section{Table 1:}

\begin{tabular}{|l|l|l|l|l|}
\hline Values for $\mathrm{n}$ & Values for $\mathrm{K}_{1}$ & $\mathrm{R}_{\text {BOUNDARY }}[\mathrm{m}]$ & Frequency $\omega_{0}$ & Area \\
\hline-10 & 1 & 0.000724227 & $2.6 \times 10^{12}$ & Electromagnetic \\
\hline
\end{tabular}




\begin{tabular}{|c|c|c|c|c|}
\hline-4 & 1 & $5.5940239 \times 10^{-10}$ & $3.37 \times 10^{18}$ & \multirow{7}{*}{$\begin{array}{l}\text { Particle } \\
\text { (Electromagnetically } \\
\text { Controlled) }\end{array}$} \\
\hline-2 & 1 & $6.7798267 \times 10^{-28}$ & $2.78 \times 10^{36}$ & \\
\hline-2 & 2 & $1.3559653 \times 10^{-27}$ & $1.39 \times 10^{36}$ & \\
\hline-2 & $10^{18}$ & $6.7798267 \times 10^{-10}$ & $2.78 \times 10^{18}$ & \\
\hline-1.6 & 1 & $3.0622921 \times 10^{-45}$ & $6.15 \times 10^{53}$ & \\
\hline-1.51 & 1 & $4.7888625 \times 10^{-52}$ & $3.94 \times 10^{60}$ & \\
\hline-1.51 & $10^{18}$ & $9.4264902 \times 10^{-17}$ & $2.0 \times 10^{25}$ & \\
\hline-1 & 1 & Infinite & & Solution of (5) \\
\hline-0.9 & 1 & $1.2126075 \times 10^{267}$ & $1.55 \times 10^{-258}$ & \multirow{4}{*}{$\begin{array}{l}\text { Electromagnetic } \\
\text { Black Hole } \\
\text { (Gravitationally } \\
\underline{\text { Controlled) }}\end{array}$} \\
\hline-0.5 & 1 & $2.1755186 \times 10^{54}$ & $8.66 \times 10^{-46}$ & \\
\hline-0.5 & $10^{18}$ & & & \\
\hline-0.5 & $10^{36}$ & & & \\
\hline
\end{tabular}

\section{Confined Electromagnetic Radiation within a Toroidal Coordinate System}

The Toroidal Coordinate System $\{\theta, r, \varphi, R, t\}$ is parameterized by the large radius $\mathrm{R}$ of the Torus. The Toroidal Coordinate System is obtained by rotating bipolar coordinates $\{r, \varphi\}$ around an axis perpendicular to the axis connecting the two foci.

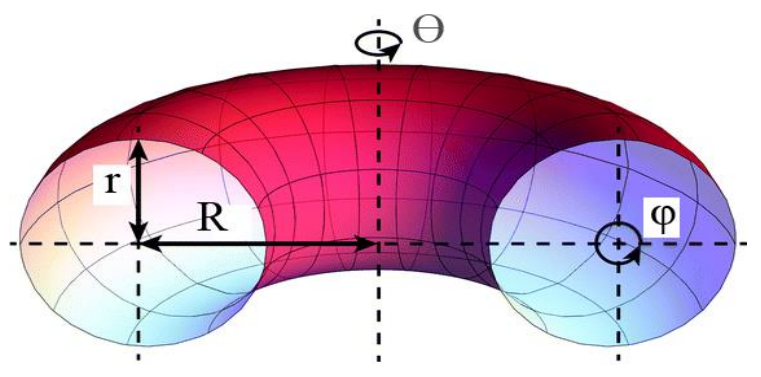
The coordinate $\{\theta\}$ specifies the angle of rotation.

The Torus in the figure below has been constructed with a Radius $R=3$ and $r=1$.

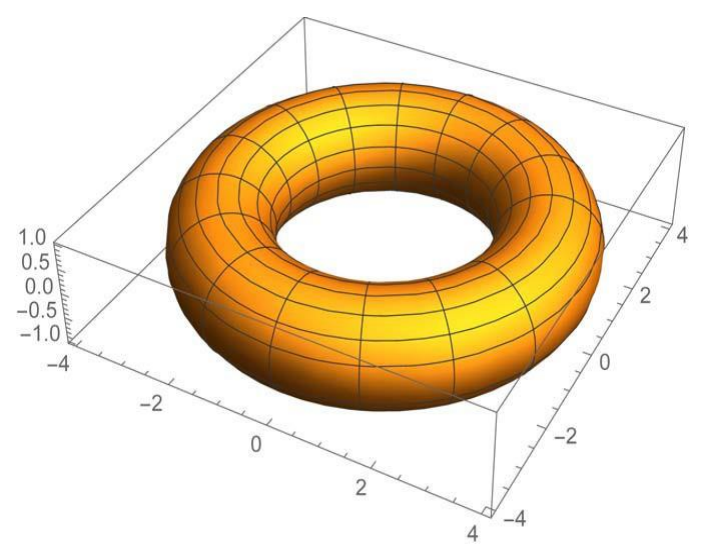


The required Electromagnetic Field Configuration for a perfect Equilibrium in Space and Time equals in Toroidal Coordinates $\{\theta, r, \varphi, t\}$ for the Electric Field Components e $(\theta, r, \varphi, t)$ :

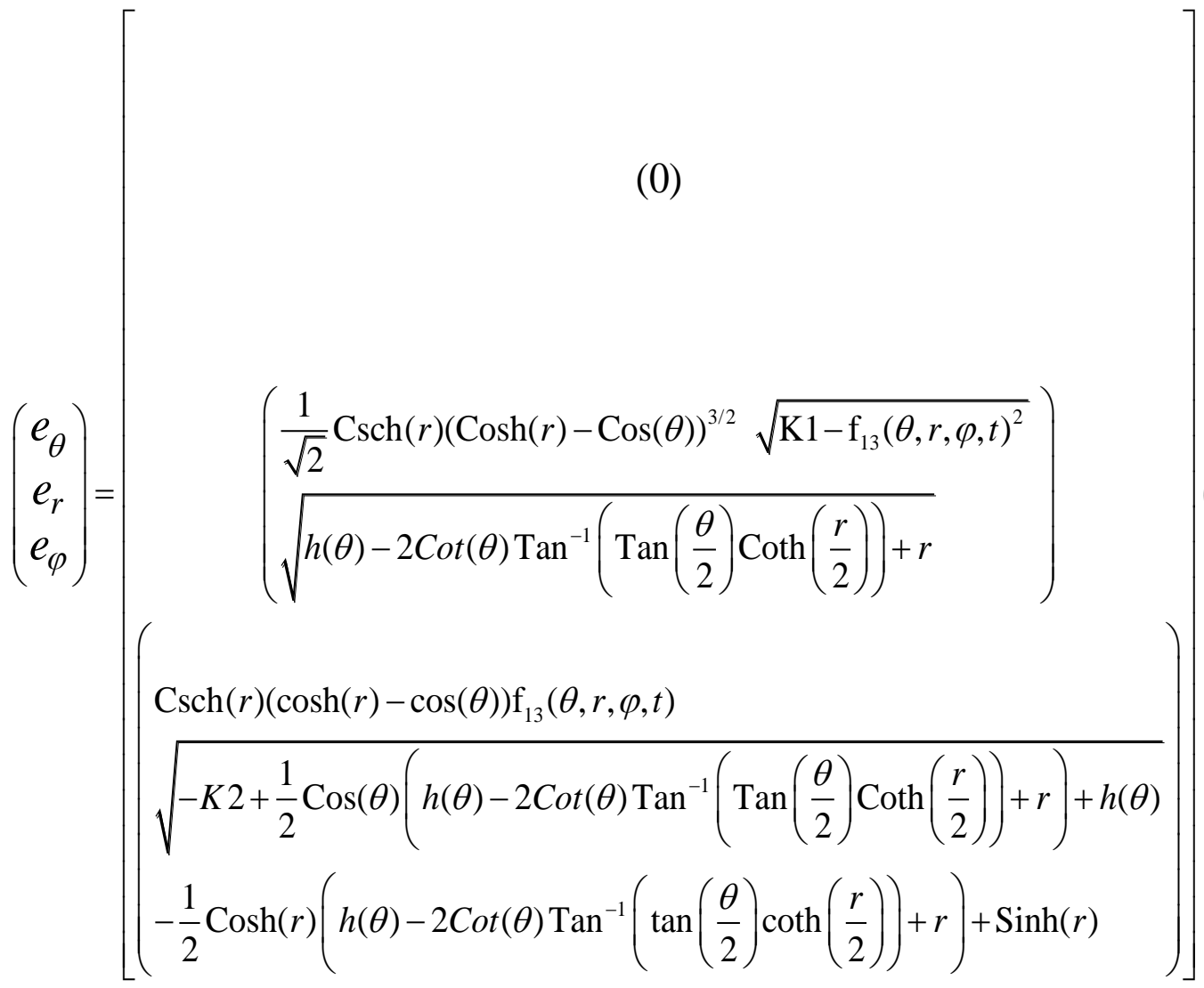

The required Electromagnetic Field Configuration for a perfect Equilibrium in Space and Time equals in Toroidal Coordinates $\{\theta, r, \varphi, t\}$ for the Magnetic Field Components $\mathrm{m}(\theta, r, \varphi, t)$ : 


$$
\left(\begin{array}{l}
m_{\theta} \\
m_{r} \\
m_{\varphi}
\end{array}\right)=\sqrt{\frac{\varepsilon_{0}}{\mu_{0}}}\left[\begin{array}{c}
(0) \\
\left(\begin{array}{c}
\frac{-1}{\sqrt{2}} \operatorname{Csch}(r)(\operatorname{Cosh}(r)-\operatorname{Cos}(\theta))^{3 / 2} \mathrm{f}_{13}(\theta, r, \varphi, t) \\
h(\theta)-2 \operatorname{Cot}(\theta) \operatorname{Tan}^{-1}\left(\operatorname{Tan}\left(\frac{\theta}{2}\right) \operatorname{Coth}\left(\frac{r}{2}\right)\right)+r
\end{array}\right) \\
\left(\begin{array}{l}
\sqrt{-K 2+\frac{1}{2} \operatorname{Cos}(\theta)\left(h(\theta)-2 \operatorname{Cot}(\theta) \operatorname{Tan}{ }^{-1}\left(\operatorname{Tan}\left(\frac{\theta}{2}\right) \operatorname{Coth}\left(\frac{r}{2}\right)\right)+r\right)+h(\theta)} \\
-\frac{1}{2} \operatorname{Cosh}(r)\left(h(\theta)-2 \operatorname{Cot}(\theta) \operatorname{Tan}^{-1}\left(\tan \left(\frac{\theta}{2}\right) \operatorname{coth}\left(\frac{r}{2}\right)\right)+r\right)+\operatorname{Sinh}(r)
\end{array}\right)
\end{array}\right]
$$

\section{Confined Electromagnetic Radiation within a Toroidal Coordinate System through} Electromagnetic-Gravitational Interaction in a non-linear Space-Time Continuum

The required Electromagnetic Field Configuration for a Gravitational-Electromagnetic Equilibrium in Space and Time equals in Toroidal Coordinates $\{\theta, r, \varphi, t\}$ for the Electric Field Components e $(\theta, r, \varphi, t)$ :

$$
\left.\left(\begin{array}{c}
e_{\theta} \\
e_{r} \\
e_{\varphi}
\end{array}\right)=\left[\begin{array}{c}
(0) \\
\left(\operatorname{Csch}(\mathrm{r}) \mathrm{f} 1(\theta, \mathrm{r})(\operatorname{Cosh}(\mathrm{r})-\operatorname{Cos}(\theta))^{3 / 2} \sqrt{\mathrm{K} 1-\mathrm{f} 13(\theta, \mathrm{r}, \varphi, \mathrm{t})^{2}}\right.
\end{array}\right)\right]
$$


The required Electromagnetic Field Configuration for a Gravitational-Electromagnetic Equilibrium in Space and Time equals in Toroidal Coordinates $\{\theta, r, \varphi, t\}$ for the Magnetic Field Components $\mathrm{m}(\theta, r, \varphi, t)$ :

$$
\left(\begin{array}{c}
m_{\theta} \\
m_{r} \\
m_{\varphi}
\end{array}\right)=\sqrt{\frac{\varepsilon_{0}}{\mu_{0}}}\left[\begin{array}{c}
(0) \\
\left(\operatorname{Csch}(\mathrm{r}) \mathrm{f} 1(\theta, \mathrm{r})\left(-(\operatorname{Cosh}(\mathrm{r})-\operatorname{Cos}(\theta))^{3 / 2}\right) \mathrm{f} 13(\theta, \mathrm{r}, \varphi, \mathrm{t})\right) \\
\left(\begin{array}{l}
\operatorname{Csch}(\mathrm{r})(\operatorname{Cosh}(\mathrm{r})-\operatorname{Cos}(\theta)) \\
\sqrt{\operatorname{Cos}(\theta) \mathrm{f} 1(\theta, \mathrm{r})^{2}-\operatorname{Cosh}(\mathrm{r}) \mathrm{f} 1(\theta, \mathrm{r})^{2}-\mathrm{g}(\mathrm{r})} \sqrt{\mathrm{K} 1-\mathrm{f} 13(\theta, \mathrm{r}, \varphi, \mathrm{t})^{2}}
\end{array}\right)
\end{array}\right](24)
$$

The toroidal electromagnetic field configuration is in perfect equilibrium with itself and its surrounding in respectively the $\theta$ - and the $\varphi$ - direction. There is a resulting electromagnetic outward bounding force density in the r-direction, $\vec{f}(\theta, r, \varphi, t)$ indicated as the outward bounding radiation pressure of the toroidal electromagnetic confinement.

$$
\vec{f}(\theta, r, \varphi, t)=\left[\begin{array}{c}
0 \\
-\frac{\varepsilon_{0} \mathrm{~K}_{1} \operatorname{Csch}^{2}(\mathrm{r})(\operatorname{Cos}(\theta)-\operatorname{Cosh}(\mathrm{r}))^{3}\left(4 \mathrm{fl}(\theta, \mathrm{r}) \mathrm{f} \mathrm{f}^{(0.1)}(\theta, \mathrm{r})(\operatorname{Cos}(\theta)-\operatorname{Cosh}(\mathrm{r}))-\mathrm{g}^{\prime}(\mathrm{r})\right)}{2 \mathrm{R}} \\
0
\end{array}\right](25)
$$

This resulting outward bounding radiation pressure has to be compensated by the inward bounding gravitational force density, to create the required equilibrium by electromagneticgravitational interaction.

In a comparable way as in the example presented in spherical coordinates in (13) and (14), the electromagnetic mass density from the energy density in the torus can be calculated. With these values the inward bounded gravitational radiation pressure can be derived. From the requirement that both force densities have to compensate each other, the Boundary Radius $R_{\text {BOUNDARY }}$ for the toroidal confinement can be calculated. 


\section{Newton's second law for Confined Electromagnetic radiation}

When Erwin Schrödinger published in 1926 the well-known Schrödinger wave equation with the characteristic spherical and elliptical wave solutions, he found a mathematical presentation for "De Broglie" or the Material Waves. When Bohr assigned these "De Broglie" waves as "Probability Waves", he excluded "De Broglie" from any mass. The mass was for $100 \%$ in the elementary particle itself and "De Broglie" waves without any mass described the probability of the position of this elementary particle. A complete non-logical approach.

A fundamental logical approach is to assign the mass to "De Broglie" waves. And that is only possible when we "De Broglie" waves are just "Confined Single Harmonic Electromagnetic Waves" (confined light). For this reason the mass of confined electromagnetic radiation has to be calculated. It will be clear that it is impossible to assign mass to free electromagnetic radiation. Because it is impossible to accelerate free electromagnetic radiation and the concept of mass if based on the inertia of mass by Newton's second law. For this reason the inertia of "Confined Single Harmonic Electromagnetic Waves" (confined light) has to be calculated.

For the first step in this calculation, an imaginary experiment has been used. Two $100 \%$ reflecting mirrors $B$ and $A$ (both in the $x-y$ plane and without any mass) are placed opposite each other at a distance $\Delta x$ ( ref 29, page 7, figure 1$)$. A single harmonic electromagnetic wave has been confined between both mirrors. Between both mirrors a "Standing Electromagnetic Wave" appears which has been formed by two waves traveling in opposite directions along the z-axis.

The Poynting vector corresponding with the electromagnetic wave propagating along the $z$-axis in the + direction (positive direction of the z-axis) has been indicated as $\overrightarrow{\mathrm{S}}^{+}=\overrightarrow{\mathrm{E}}^{+} \times \overrightarrow{\mathrm{H}}^{+}$and the Poynting vector corresponding with the electromagnetic wave propagating along the $z$-axis in the - direction (opposite direction) has been indicated as $\overrightarrow{\mathrm{S}}^{-}=\overrightarrow{\mathrm{E}}^{-} \times \overrightarrow{\mathrm{H}}^{-}$.

The system is at rest. The radiation pressures, caused by the confined electromagnetic radiation, on both mirrors $A$ and $B$ are opposite and equal in magnitude:

$$
\mathrm{P}_{\mathrm{A}}=\frac{2 \mathrm{~S}_{\mathrm{A}}}{\mathrm{c}}=\frac{2 \mathrm{~S}_{\mathrm{B}}}{\mathrm{c}}=\mathrm{P}_{\mathrm{B}}
$$

Einstein has formulated this very well. "Velocities are always relative" . To calculate the radiation pressure on Mirror A, the velocities, only relative to Mirror A for the waves with the respective Poynting vectors $\overrightarrow{\mathrm{S}}^{+}=\overrightarrow{\mathrm{E}}^{+} \times \overrightarrow{\mathrm{H}}^{+}$and $\overrightarrow{\mathrm{S}}^{-}=\overrightarrow{\mathrm{E}}^{-} \times \overrightarrow{\mathrm{H}}^{-} \mathrm{M}$, have to be calculated. 


\subsubsection{The radiation pressure on Mirror $A$, when Mirror A moves with a velocity $v$ in the} direction of the positive $z$-axis

When the system of "Two Mirrors B $\longleftrightarrow A$ " moves in the direction of the positive z-axis, Mirror A moves in the direction of the positive z-axis and the Poynting vector $\overrightarrow{\mathrm{S}}^{+}=\overrightarrow{\mathrm{E}}^{+} \times \overrightarrow{\mathrm{H}}^{+}$will decrease according to the Lorentz transformation ( ref. 29, page 23, A-57).

$\overrightarrow{\mathrm{S}}_{\mathrm{v}}^{+}=\overrightarrow{\mathrm{E}}_{\mathrm{v}}^{+} \times \overrightarrow{\mathrm{H}}_{\mathrm{v}}^{+}=\gamma^{2}\left(1-\frac{\mathrm{v}}{\mathrm{c}}\right)^{2}\left(\overrightarrow{\mathrm{E}}^{+} \times \overrightarrow{\mathrm{H}}^{+}\right)$

When the system of "Two Mirrors B $\longleftrightarrow A$ " moves in the direction of the positive z-axis, Mirror A moves in the direction of the positive z-axis the Poynting vector $\overrightarrow{\mathrm{S}}^{-}=\overrightarrow{\mathrm{E}}^{-} \times \overrightarrow{\mathrm{H}}^{-}$will increase according to the Lorentz transformation ( ref. 29, page 23, A-57 ).

$\overrightarrow{\mathrm{S}}_{\mathrm{v}}^{-}=\overrightarrow{\mathrm{E}}_{\mathrm{v}}^{-} \times \overrightarrow{\mathrm{H}}_{\mathrm{v}}^{-}=\gamma^{2}\left(1+\frac{\mathrm{v}}{\mathrm{c}}\right)^{2}\left(\overrightarrow{\mathrm{E}}^{+} \times \overrightarrow{\mathrm{H}}^{+}\right)$

The total radiation pressure, caused by the confined electromagnetic radiation, on mirror $\mathrm{A}$ equals:

$\mathrm{P}_{\mathrm{A}}=\frac{\mathrm{S}_{A}^{+}+\mathrm{S}_{A}^{-}}{\mathrm{c}}=\frac{\gamma^{2}\left(\left(1-\frac{\mathrm{v}}{\mathrm{c}}\right)^{2}+\left(1+\frac{\mathrm{v}}{\mathrm{c}}\right)^{2}\right)\left(\overrightarrow{\mathrm{E}}^{+} \times \overrightarrow{\mathrm{H}}^{+}\right)}{\mathrm{c}}$

9.1.2 The radiation pressure on Mirror B when Mirror B moves with a velocity $v$ in the direction of the positive $z$-axis

When the system of "Two Mirrors B $\longleftrightarrow A$ " moves in the direction of the positive z-axis, Mirror $B$ moves in the direction of the positive z-axis and the Poynting vector $\overrightarrow{\mathrm{S}}^{-}=\overrightarrow{\mathrm{E}}^{-} \times \overrightarrow{\mathrm{H}}^{-}$will increase according to the Lorentz transformation ( ref. 29, page 23, A-57 ).

$\overrightarrow{\mathrm{S}}_{\mathrm{v}}^{-}=\overrightarrow{\mathrm{E}}_{\mathrm{v}}^{-} \times \overrightarrow{\mathrm{H}}_{\mathrm{v}}^{-}=\gamma^{2}\left(1+\frac{\mathrm{v}}{\mathrm{c}}\right)^{2}\left(\overrightarrow{\mathrm{E}}^{+} \times \overrightarrow{\mathrm{H}}^{+}\right)$

When the system of "Two Mirrors $B \longleftrightarrow A$ " moves in the direction of the positive z-axis, Mirror A moves in the direction of the positive z-axis the Poynting vector $\overrightarrow{\mathrm{S}}^{+}=\overrightarrow{\mathrm{E}}^{+} \times \overrightarrow{\mathrm{H}}^{+}$will increase according to the Lorentz transformation ( ref. 29, page 23, A-57 ). 
$\overrightarrow{\mathrm{S}}_{\mathrm{v}}^{+}=\overrightarrow{\mathrm{E}}_{\mathrm{v}}^{+} \times \overrightarrow{\mathrm{H}}_{\mathrm{v}}^{+}=\gamma^{2}\left(1-\frac{\mathrm{v}}{\mathrm{c}}\right)^{2}\left(\overrightarrow{\mathrm{E}}^{+} \times \overrightarrow{\mathrm{H}}^{+}\right)$

The total radiation pressure, caused by the confined electromagnetic radiation, on mirror B equals:

$$
\mathrm{P}_{\mathrm{A}}=\frac{\mathrm{S}_{A}^{+}+\mathrm{S}_{A}^{-}}{\mathrm{c}}=\frac{\gamma^{2}\left(\left(1+\frac{\mathrm{v}}{\mathrm{c}}\right)^{2}+\left(1-\frac{\mathrm{v}}{\mathrm{c}}\right)^{2}\right)\left(\overrightarrow{\mathrm{E}}^{+} \times \overrightarrow{\mathrm{H}}^{+}\right)}{\mathrm{c}}
$$

$\mathrm{P}_{\mathrm{A}}$ and $\mathrm{P}_{B}$ are still equal in magnitude and both in opposite direction and still cancel each other. The system fulfils Newton's first law of motion.

When the system of "Two Mirrors $\mathrm{B} \longleftrightarrow A$ " accelerates, the velocity increases with $\Delta \mathrm{v}$ in a time interval $\Delta \mathrm{t}$. At time $\mathrm{t}$ the radiation pressures on mirror $\mathrm{A}$ and mirror $\mathrm{B}$ are presented in (29) and (32). At time $t+\Delta t$ the radiation pressures on Mirror $A$ and Mirror $B$ will different:

The radiation pressure at time $t+\Delta t$ caused by the confined electromagnetic radiation, on mirror A equals:

$$
\mathrm{P}_{\mathrm{A}}=\frac{\mathrm{S}_{A}^{+}+\mathrm{S}_{A}^{-}}{\mathrm{c}}=\frac{\gamma^{2}\left(\left(1+\frac{(\mathrm{v})}{\mathrm{c}}\right)^{2}+\left(1-\frac{(\mathrm{v}+\Delta \mathrm{v})}{\mathrm{c}}\right)^{2}\right)\left(\overrightarrow{\mathrm{E}}^{+} \times \overrightarrow{\mathrm{H}}^{+}\right)}{\mathrm{c}}
$$

Because the wave with Poynting vector $\overrightarrow{\mathrm{S}}^{+}=\overrightarrow{\mathrm{E}}^{+} \times \overrightarrow{\mathrm{H}}^{+}$has left Mirror $\mathrm{B}$ at " $\mathrm{t}$ " and during the time interval $\Delta \mathrm{t}$ the magnitude of $\overrightarrow{\mathrm{E}}_{\mathrm{t}}^{+}=\left(1+\frac{\mathrm{v}}{\mathrm{c}}\right) \overrightarrow{\mathrm{E}}^{+}$and $\overrightarrow{\mathrm{H}}_{\mathrm{t}}^{+}=\left(1+\frac{\mathrm{v}}{\mathrm{c}}\right) \overrightarrow{\mathrm{H}}^{+}$has not changed.

The radiation pressure at time $\mathrm{t}+\Delta \mathrm{t}$ caused by the confined electromagnetic radiation, on mirror $B$ equals:

$$
\mathrm{P}_{B}=\frac{\mathrm{S}_{B}^{+}+\mathrm{S}_{B}^{-}}{\mathrm{c}}=\frac{\gamma^{2}\left(\left(1+\frac{(\mathrm{v}+\Delta \mathrm{v})}{\mathrm{c}}\right)^{2}+\left(1-\frac{(\mathrm{v})}{\mathrm{c}}\right)^{2}\right)\left(\overrightarrow{\mathrm{E}}^{+} \times \overrightarrow{\mathrm{H}}^{+}\right)}{\mathrm{c}}
$$

Because the wave with Poynting vector $\overrightarrow{\mathrm{S}}^{-}=\overrightarrow{\mathrm{E}}^{-} \times \overrightarrow{\mathrm{H}}^{-}$has left Mirror $\mathrm{A}$ at " $\mathrm{t}$ " and during the time interval $\Delta \mathrm{t}$ the magnitude of $\overrightarrow{\mathrm{E}}_{\mathrm{t}}^{-}=\left(1+\frac{\mathrm{v}}{\mathrm{c}}\right) \overrightarrow{\mathrm{E}}^{-}$and $\overrightarrow{\mathrm{H}}_{\mathrm{t}}^{-}=\left(1+\frac{\mathrm{v}}{\mathrm{c}}\right) \overrightarrow{\mathrm{H}}^{-}$has not changed. 
The radiation pressures on Mirror A and Mirror B do not counterbalance each other anymore and the resulting radiation pressure equals:

$$
\mathrm{P}_{\mathrm{B}}-\mathrm{P}_{\mathrm{A}}=\frac{\gamma^{2}(4 \Delta \mathrm{v}) \mathrm{S}}{\mathrm{c}^{2}}
$$

Equation (35) can be written as:

$$
\mathrm{P}_{\mathrm{B}}-\mathrm{P}_{\mathrm{A}}=\frac{\gamma^{2}(4 \Delta \mathrm{v}) \mathrm{S}}{\mathrm{c}^{2}}=\frac{\gamma^{2}\left(4 \frac{\Delta \mathrm{v}}{\Delta \mathrm{t}}\right) \mathrm{S} \Delta \mathrm{t}}{\mathrm{c}^{2}}=\gamma^{2} \frac{\mathrm{W}}{\mathrm{c}^{2}} \mathrm{a}=\gamma^{2} \mathrm{ma}
$$

In which the acceleration $\mathrm{a}=\frac{\Delta \mathrm{v}}{\Delta \mathrm{t}}$ and the inertia $\mathrm{m}=\frac{\mathrm{W}}{\mathrm{c}^{2}}$. At non-relativistic velocities $\gamma=1$ and (36) turns into the Newton's second law of motion. From (36) also Einstein's famous equation $\mathrm{W}=\mathrm{m} \mathrm{c}^{2}$ follows. In (36) $\mathrm{W}$ is the total confined electromagnetic mass.

Now we can consider electromagnetic confinements without mirrors but electromagnetic confinements through electromagnetic gravitational interaction. By superposition and integration over arbitrary surfaces, it is possible to prove that all confined electromagnetic radiation equals (36) and has electromagnetic mass and follow Newton's second law of motion.

\section{Concluding Remarks}

The example of Gravitational-Electromagnetic Interaction presented in table 1 shows two types of confinement.

1. For values $0<\mathrm{n}<-1$, the Gravitational-Electromagnetic Confinement will be Gravitationally controlled (Table 1). This means that for values for $r>R_{\text {BOUNDARY }}$ the inward bounded Gravitational for will be larger than the outward bounded Electromagnetic Radiation pressure. Electromagnetic Radiation will be attracted by Gravity towards the confinement at the surface $R_{\text {BOUNDARY }}$. Because for values $r<R_{\text {BOUNDARY }}$ the outward bounded radiation pressure is higher than the inward bounded gravitational pressure, all the radiation will be forced to be confined at equilibrium just at the surface of the spherical sphere with radius $\mathrm{R}_{\text {BOUNDARY }}$. The confinement can be considered as an Electromagnetic Black Hole.

2. For values $-1<\mathrm{n}<-\infty$, the Gravitational-Electromagnetic Confinement will be Electromagnetically controlled (Table 1). This means that for values for $r>R_{\text {BoundARY }}$ the inward bounded Gravitational for will be smaller than the outward bounded Electromagnetic Radiation pressure. Electromagnetic Radiation will be scattered by the 
Radiation Pressure away from the confinement at the surface $R_{\text {BOUNDARY }}$. Because for values $r<R_{\text {BOUNDARY }}$ the outward bounded radiation pressure is smaller than the inward bounded gravitational pressure, all the radiation will be confined within the sphere with radius $\mathrm{R}_{\text {BOUNDARY }}$. The confinement can be considered as an Electromagnetic Particle.

3. For values $n=-1$, the inward bounded Gravitational pressure equals the outward bounded Electromagnetic Radiation pressure at any distance $r$. The calculated value for $\mathrm{R}_{\text {BOUNDARY }}$ becomes $\mathrm{R}_{\text {BOUNDARY }} \rightarrow \infty$.

Because of the extremely high-energy densities within electromagnetic-gravitational confinements and the extremely small dimensions, the radiation pressure at small densities will be extremely high. For this reason, single harmonic (monochromatic) electromagneticgravitational confinements will behave like nondeformable particles in experiments.

Now the four Castles have fallen:

1) The Castle of the Dice. It follows from (36) that "Confined Single Harmonic Electromagnetic Waves" are far superior candidates for "De Broglie" waves because they carry the mass (electromagnetic mass) and probability waves do not carry mass at all.

After the first publications of the famous first photo of the hydrogen atom, it became clear that what had been photographed could never be a photo of a non-existing complex probability wave with zero mass. And it will be clear that due to Electromagnetic Interaction it is very well possible to photograph a confined monochromatic electromagnetic wave. Instead of the nonlogical model of a zero mass probability wave describing the probability that the mass of an elementary particle is at a certain time at a certain place, it is only logical to define the mass division through the electromagnetic mass division of a "Confined Single Harmonic Electromagnetic Wave" according Equation (36).

For that reason it has been concluded that "De Broglie" waves are not complex probability waves but are "Confined Single Harmonic Electromagnetic Waves" written in a complex way. (Equation 13, Ref.29) ) like the example of a confined single harmonic electromagnetic wave in Equation (13) in this manuscript.

2) The Castle of Schrödinger. The Schrödinger wave equation is nothing more than the Continuity Equation (equation 42, Ref.29 ) written in a complex way (equation 55, Ref.29).

3) The Castle of Dirac. The relativistic Dirac Equation is nothing more than the Continuity Equation (equation 57, Ref.29) written in a complex way (equation 102, Ref.29). 
4) The Castle of Maxwell. The 4 Maxwell Equations are incomplete. The correct equation is Equation (5) in this manuscript or (Equation 3, Ref 30).

Now the four castles have fallen, there is only one possible final conclusion: Indeed, God does not play Dice. Probability does not exist. And without probability, elementary particles cannot exist. Light (Confined Single Harmonic Electromagnetic Radiation) is the building element of our universe. As Jesus has spoken: "I am the Light of the World (John 8:12). He spoke for the world. Now the world is free to leave the gambling house and to stop playing dice with the devil. Instead of spending trillions of dollars to investigate non-existing elementary particles in extremely expensive high energy accelerators, the world has the possibility to spend trillions of dollars to create, to build and to improve 'drinking water systems', that change ocean water into drinking water, to turn the deserts into oases of green, into oases of fruit trees and vegetables. Instead of killing warzones, the whole Mideast could turn into an oasis of prosperity where everybody has enough to eat and these countries will have the possibilities to export fruit and vegetables. Turn whole Africa into an Oasis of green. You have the power and you can do it. Now the world will have the possibilities to spend trillions of dollars to build dikes to protect the people in the dangerous lowland that will come underwater when the ocean levels are rising. Science is given to mankind to serve the people and to make their lives better.

\section{Acknowledgment}

This work has been made possible by the contributions of many scientists who have given their life to find a path of truth. I specially would like to thank (in memory of) Professor Dr. F.W. Sluijter TU/e, The Netherlands, who has shown me the way to physics that has been forgotten by so many. A humble man with a great wisdom. For me, he has always been one of the happy few of the superior level of Albert Einstein who thinks completely independently. I am indebted to all and to my family, who always has supported me on a long and insecure road.

In particular, I would like to thank Dr. Albert Einstein, an honest man of wisdom, independent thinker and a great insight in Physics. He had the insight to see that the 'Scientific World' was going to make the biggest mistake in History at the 1927 Solvay Conference where Bohr's concept of 'Probability Waves' was generally accepted. And he warned the World: 'God does not play Dice'. And after 100 years, his words are still echoing through the world. And they will

never stop echoing until the 'Scientific World' wakes up from the dream of the 'Dice that Rules the Universe'.

\section{References}

1. J. Wheeler, Phys. Rev. 97, 511 (1955).

2. W.G.V. Rosser, Classical Electromagnetism via Relativity (Butterworths, London, 1968), p. 134. 
3. Brando Bellazzini, Francesco Riva, Javi Serra, and Francesco Sgarlata; Beyond Positivity Bounds and the Fate of Massive Gravity ; Phys. Rev. Lett. 120, 161101 - Published 17 April 2018

4. Bao-Fei Li, Parampreet Singh, and Anzhong Wang; Towards cosmological dynamics from loop quantum gravity ;Phys. Rev. D 97, 084029 - Published 17 April 2018

5. L. Filipe O. Costa, Georgios Lukes-Gerakopoulos, and Oldřich Semerák; Spinning particles in general relativity: Momentum-velocity relation for the Mathisson-Pirani spin condition; Phys. Rev. D 97, 084023 - Published 16 April 2018

6. Ryotaro Kase, Masato Minamitsuji, and Shinji Tsujikawa; Relativistic stars in vector-tensor theories, Phys. Rev. D 97, 084009 - Published 9 April 2018

7. Hector O. Silva, Jeremy Sakstein, Leonardo Gualtieri, Thomas P. Sotiriou, and Emanuele Berti; Spontaneous Scalarization of Black Holes and Compact Stars from a Gauss-Bonnet Coupling; Phys. Rev. Lett. 120, 131104 (2018) - Published 30 March 2018

8. Jahed Abedi, Hannah Dykaar, and Niayesh Afshordi; Echoes from the abyss: Tentative evidence for Planck-scale structure at black hole horizons ;Phys. Rev. D 96, 082004 (2017) - Published 26 October 2017

9. A. Hees, T. Do, A. M. Ghez, G. D. Martinez, S. Naoz, E. E. Becklin, A. Boehle, S. Chappell, D. Chu, A. Dehghanfar, K. Kosmo, J. R. Lu, K. Matthews, M. R. Morris, S. Sakai, R. Schödel, and G. Witzel; Testing General Relativity with Stellar Orbits around the Supermassive Black Hole in Our Galactic Center; Phys. Rev. Lett. 118, 211101 (2017) - Published 25 May 2017

10. Petr Hořava ; Spectral Dimension of the Universe in Quantum Gravity at a Lifshitz Point; Phys. Rev. Lett. 102, 161301 (2009); Published April 20, 2009

11. Kenji Hayashi and Takeshi Shirafuji ; Addendum to 'New general relativity' ; Phys. Rev. D 24, 3312 - Published 15 December 1981

12 Talmadge M. Davis and John R. Ray ; Ghost neutrinos in general relativity; Phys. Rev. D 9, 331 (1974) - Published 15 January 1974

13 Patrick G. Whitman and Richard C. Burch ; Charged spheres in general relativity; Phys. Rev. D 24, 2049 - Published 15 October 1981; Erratum Phys. Rev. D 25, 1744 (1982)

14 Joseph Jacobson, Gunnar Björk, Isaac Chuang, and Yoshihisa Yamamoto; Photonic de Broglie Waves ; Phys. Rev. Lett. 74, 4835 (1995) - Published 12 June 1995

15 Bogeun Gwak and Bum-Hoon Lee ; Instability of rotating anti-de Sitter black holes ; Phys. Rev. D 91, 064020 - Published 9 March 2015

16 Raphael Bousso and Stephen Hawking ; Erratum: Lorentzian condition in quantum gravity [Phys. Rev. D 59, 103501 (1999)] ; Phys. Rev. D 60, 109903 (1999) - Published 8 October 1999

17 A. Steane, P. Szriftgiser, P. Desbiolles, and J. Dalibard ; Phase Modulation of Atomic de Broglie Waves ; Phys. Rev. Lett. 74, 4972 - Published 19 June 1995

18 S. B. Cahn, A. Kumarakrishnan, U. Shim, T. Sleator, P. R. Berman, and B. Dubetsky ; Time-Domain de Broglie Wave Interferometry ; Phys. Rev. Lett. 79, 784 - Published 4 August 1997

19 J. L. Synge ; Primitive Quantization in the Relativistic Two-Body Problem ; Phys. Rev. 89, 467 Published 15 January 1953

20 H. Jehle, Flux Quantization and particle Physics, Phys. Rev. D6 (1972) $441-457$ 
21 Osung Kwon, Young-Sik Ra, and Yoon-Ho Kim ; Observing photonic de Broglie waves without the maximally-path-entangled $|\mathrm{N}, 0\rangle+|0, N\rangle$ state ; Phys. Rev. A 81, 063801 (2010) - Published 1 June 2010

22 V. Krachmalnicoff, J.-C. Jaskula, M. Bonneau, V. Leung, G. B. Partridge, D. Boiron, C. I. Westbrook, P. Deuar, P. Ziń, M. Trippenbach, and K. V. Kheruntsyan ; Spontaneous Four-Wave Mixing of de Broglie Waves: Beyond Optics ; Phys. Rev. Lett. 104, 150402 (2010) - Published 15 April 2010

23 Andrey Turlapov, Alexei Tonyushkin, and Tycho Sleator ; Talbot-Lau effect for atomic de Broglie waves manipulated with light ; Phys. Rev. A 71, 043612 (2005) - Published 25 April 2005

Jakob Petersen, Eli Pollak, and Salvador Miret-Artes ; Alberto Nicolis and Riccardo Penco ; Mutual interactions of phonons, rotons, and gravity; Phys. Rev. B 97, 134516 (2018) - Published 18 April 2018

Quantum threshold reflection is not a consequence of a region of the long-range attractive potential with rapidly varying de Broglie wavelength ; Phys. Rev. A 97, 042102 (2018) - Published 3 April 2018

26 Jing, H., Jiang, Y. \& Deng, Y.; Quantum superchemistry of de Broglie waves: New wonderland at ultracold temperature ; Front. Phys. China (2011) 6: 15. https://doi.org/10.1007/s11467010-0155-y

27 Donald H Kobe ; Quantum power in de Broglie-Bohm theory ; Journal of Physics A: Mathematical and Theoretical, Volume 40 - Number 19, Published 24 April 2007

28 Rodewald, J., Haslinger, P., Dörre, N. et al.; New avenues for matter-wave-enhanced spectroscopy ; Appl. Phys. B (2017) 123: 3. https://doi.org/10.1007/s00340-016-6573-y

29. J. W. Vegt, A Continuous Model of Matter based on AEONs, Physics Essays,1995, Volume 8, Number 2,

201-224 A Continuous model of Matter (DOI: 10.13140/RG.2.2.25149.77281).

30. J. W. Vegt, Annales Fondation Louis de Broglie, The Maxwell-Schrödinger-Dirac Correspondence in Auto

Confined Electromagnetic Fields, Annales Fondation Louis de Broglie, 2002, Volume 27, Number 1 ,

31. J. W. Vegt, A particle Free Model of Matter based on Electromagnetic Self-Cofinement, Annales Fondation

Louis de Broglie, 1996, January.

32. J. M. Maldacena, Black Holes in String Theory, Princeton University, arxiv.org/abs/hepth/960723533.

33. V. C. de Andrade and J. G. Pereira, Gravitational Lorentz force and the description of the gravitational interaction, Phys. Rev. D 56, 468

34. Mohr, P.J.; Taylor, B.N.; Newell, D.B. (2006). 'CODATA recommended values of the fundamental physical constants'. Reviews of Modern Physics. 80(2): 633-730. arXiv:0801.0028, Bibcode:2008RvMP...80..633M. doi:10.1103/RevModPhys.80.633.

35. J. M. Maldacena, Black Holes in String Theory, Princeton University, arxiv.org/abs/hepth/960723533.

36. V. C. de Andrade and J. G. Pereira, Gravitational Lorentz force and the description of the gravitational interaction, Phys. Rev. D 56, 468 
37. Mohr, P.J.; Taylor, B.N.; Newell, D.B. (2006). 'CODATA recommended values of the fundamental physical constants'. Reviews of Modern Physics. 80(2): 633-730. arXiv:0801.0028, Bibcode:2008RvMP...80..633M. doi:10.1103/RevModPhys.80.633.

38. William A. Hiscock, Phys. Rev. D 31, 3288 - Published 15 June 1985

39. T. Degrand, L. Jaffe, K Johnson, J. Kiskis, Masses and other parameters of the light Hadrons, Physical Review D: Particles and Fields, 12(7), October 1975, 2060-2076

40 Volodymyr Krasnoholovets, Motion of a Relativistic Particle and the Vacuum, Physics Essays, vol. 10, No. 3, 1997, 407-416, arXiv:quant-ph/9903077

41 H. Jehle, Flux Quantization and fractional charges of quarks, Phys. Rev. D11(1975) 2147 